\title{
Coffee brew intake can prevent the reduction of lens glutathione and ascorbic acid levels in HFD-fed animals
}

\author{
YOSUKE NAKAZAWA ${ }^{1}$, NANA ISHIMORI $^{1}$, JUN OGUCHI $^{1}$, NORIAKI NAGAI $^{2}$, \\ MASAKI KIMURA $^{3}$, MEGUMI FUNAKOSHI-TAGO ${ }^{1}$ and HIROOMI TAMURA ${ }^{1}$ \\ ${ }^{1}$ Department of Hygienic Chemistry, Faculty of Pharmacy, Keio University, Tokyo 105-8512; \\ ${ }^{2}$ Department of Advanced Design for Pharmaceuticals, Faculty of Pharmacy, Kindai University, Osaka 577-8502; \\ ${ }^{3}$ Division of Pharmacotherapeutics, Faculty of Pharmacy, Keio University, Tokyo 105-8512, Japan
}

Received July 6, 2018; Accepted December 5, 2018

DOI: $10.3892 /$ etm.2018.7092

\begin{abstract}
The lens has high concentrations of glutathione (GSH) and ascorbic acid (AsA) to maintain redox activity and prevent cataract formation, which is the leading cause of visual impairment worldwide. Metabolic syndrome is reported to be linked with a higher risk of age-associated cataract. As it was demonstrated previously that coffee consumption improved high-fat diet (HFD) -induced metabolic symptoms, it was hypothesized that coffee intake could delay the onset of obesity related-cataract; however, the effect of coffee consumption on this type of cataract remains unknown. Four-week-old male C57BL/6JJms SLC mice were divided into two groups and were provided ad libitum access to either a control diet (control groups) or a HFD (HFD groups). The control groups and HFD groups were further divided into three or four subgroups for each experiment. Coffee intake markedly reduced the increase in body weight in a roasting-time and concentration-dependent manner. Coffee consumption also prevented the HFD-induced decrease in the concentration of GSH and AsA, and treatment with pyrocatechol or caffeine also restored the reduction of antioxidant compounds. Plasma cholesterol and triglycerides were significantly higher in HFD groups; however, coffee brew or coffee constituent treatment in the HFD-fed mice group prevented elevation of these levels. Caffeine is a major coffee component and pyrocatechol is generated thought the roasting process. These results revealed that caffeine and pyrocatechol in coffee brew may be the key constituents responsible for preventing the reduction of lens GSH and AsA in HFD-fed animals.
\end{abstract}

Correspondence to: Dr Yosuke Nakazawa, Department of Hygienic Chemistry, Faculty of Pharmacy, Keio University, 1-5-30, Shibako-en, Minato-ku, Tokyo 105-8512, Japan

E-mail: nakazawa-ys@pha.keio.ac.jp

Abbreviations: GSH, glutathione; AsA, ascorbic acid; DTNB, dithionitrobenzene; DCPIP, 2,6-dichlorophenolindophenol; HFD, high-fat diet; GSSG, glutathione disulfide/oxiglutathione

Key words: glutathione, ascorbic acid, coffee, caffeine, pyrocatechol

\section{Introduction}

Cataract, defined as opacification of the lens, is the leading cause of visual impairment worldwide. Given the extended life expectancy and an increased aging of the population, the burden and impact of age-related cataract are expected to become more significant. The lens has high concentrations of glutathione (GSH) and ascorbic acid (AsA), which act to maintain redox activity and preserve lens transparency. Given that lens opacity is a direct result of oxidative stress, the concentrations of these antioxidants are frequently used as markers of cataract formation, both clinically and in animal models $(1,2)$. As the mature fibre cells that compose the bulk of the lens lack the necessary cellular organelles required to perform de novo protein synthesis, one preferred approach for the prevention of cataract is the preservation of the redox state in the lens. Multiple pieces of evidence have suggested the consumption of antioxidant compounds as a possible prevention strategy against cataract formation (3-5). There have been reports that the risk of age-related cataract is increased by metabolic syndrome or obesity $(6,7)$.

Recently, our laboratory demonstrated that coffee consumption significantly improved metabolic symptoms in mice, including body weight reduction and the accumulation of adipose tissue (8). Owing to the fact that coffee contains over 1,000 bioactive compounds, including antioxidant, anti-inflammatory, and anti-cancer agents, the accumulation of evidence from numerous epidemiological studies has revealed that coffee consumption could lower the risk of various chronic illnesses, such as cancer, cardiovascular disease, type 2 diabetes, and cataract (9-11). The effect of coffee consumption on antioxidant activity is unlikely to be a direct consequence of the antioxidant properties of the coffee brew; they are more likely to result from certain constituents of coffee augmenting endogenous antioxidant defences, such as upregulation of GSH concentration and glutathione-S-transferase (GST) activity (12). Numerous studies have shown that consumption of coffee brew helps to mitigate cataract formation in humans and animals $(5,13,14)$. We hypothesized that coffee intake could prevent obesity related-cataracts; however, the effect of coffee consumption on this type of cataract remains unknown. To validate this hypothesis, we investigated the effect of coffee on 
obesity-related cataract development. In this study, we evaluated the anti-cataract activity of coffee consumption by using high-fat diet (HFD)-fed animals as an obesity-induced cataract model and measured the levels of GSH and AsA in the lens.

\section{Materials and methods}

Materials. C57BL/6JJms SLC mice were purchased from Sankyo Labo Service Corporation (Tokyo, Japan). The control diet (D12450J) and HFD (D1249) for mice were purchased from Research Diets Inc. (New Brunswick, NJ, USA). The quantificationkit for GSSG/GSH was purchased from Dojindo Laboratories (Kumamoto, Japan). 2,6-Dichlorophenolindophenol (DCPIP) was purchased from Merck KGaA (Darmstadt, Germany). Caffeine, GSH, dithionitrobenzene (DTNB), AsA, metaphosphoric acid, caffeine, and pyrocatechol were purchased from Wako Pure Chemical Industries, Ltd. (Osaka, Japan).

Animals. Four-week-old male C57BL/6JJms SLC mice were housed under temperature-controlled condition $\left(23 \pm 5^{\circ} \mathrm{C}\right)$ with a $12 \mathrm{~h}$ regular light/dark cycle. Mice were euthanized with isoflurane (5\%, inhalation; Wako Pure Chemical Industries Ltd.). Blood samples were immediately collected form the vena cava; as much blood as possible was obtained from each mouse for subsequent measurements. All animal experiments were conducted in accordance with the approved guidelines and the National Institutes of Health (NIH) Guide for the care and use of laboratory animals. The Keio University Animal Research Committee approved all animal procedures used in this study [12048-(3)].

Preparation of coffee extract. In this study, we have used Columbia Arabica coffee because it is one of the most consumed varieties globally. Roasted Columbia Arabica coffee beans was obtained from Starbucks Coffee Japan (Tokyo, Japan). Columbian Arabica coffee green beans were purchased from Nakaya Coffee (Tokyo, Japan). Coffee beans were freshly ground before preparing the coffee extract. The coffee extract was prepared by a common method (drip style), and divided into aliquots. Coffee concentrations were adjusted for optical density measurement such that, optical density at $500 \mathrm{~nm}$ of $10 \%$ green beans extract was 0.05 , and that of $10 \%$ roasted beans extract was 0.269 .

High-fat diet-treated mice and coffee intake. Four-week-old male C57BL/6JJms SLC mice were divided into two groups: Control diet-fed mice (control groups) and HFD-fed mice (HFD groups). During the experimental period, the control groups was fed D12450J and the HFD groups was fed D1249 for 7 weeks ad libitum. The control and HFD groups were further divided into three or four subgroups. For the roasting experiment, pure water, $60 \%$ (v/v) green beans extract, and $60 \%(\mathrm{v} / \mathrm{v})$ roasted beans extract were allowed for each group ad libitum. For coffee concentration experiment, pure water, $40 \%$ (v/v) coffee extract, and 60\% (v/v) coffee extract were allowed ad libitum ( $\mathrm{n}=6$ per group in each experiment). For the coffee component experiments, pure water, $60 \%$ (v/v) coffee extract, $3.24 \mathrm{mM}$ caffeine, and $20.4 \mu \mathrm{M}$ pyrocatechol were administered for each group; these are the equivalent concentrations in $60 \%$ coffee brew (Table I) (14).
Table I. Changes in the constituents of coffee extract during the roasting process.

\begin{tabular}{lcc}
\hline & \multicolumn{2}{c}{ Coffee beans $(\mathrm{mM})$} \\
\cline { 2 - 3 } Constituents & Green & Dark \\
\hline Caffeine & 4.4 & 5.610 \\
Chlorogenic acid & 4.8 & 0.234 \\
Pyrocatechol & n.d. & 0.034 \\
Trigonelline & 4.2 & 1.120 \\
\hline
\end{tabular}

n.d., not detected.

Measurement of GSH. The concentration of GSH in the lens was determined by using the GSSG/GSH measurement kit in accordance with the manufacturer's instructions. Briefly, lenses were homogenized in 5\% 5-sulfosalicylic acid dehydrate and centrifuged. GSH masking reagent or PBS was added to the supernatant to sequester GSH for the glutathione disulfide (GSSG) concentration or total glutathione concentration measurement, respectively, and incubated for $1 \mathrm{~h}$ at $37^{\circ} \mathrm{C}$. Ten min after the addition of DTNB, the absorbance at $415 \mathrm{~nm}$ was measured by using a microplate reader infinite M200 (Tecan Ltd., Männedorf, Switzerland).

Measurement of AsA. The levels of AsA in the lens were determined using DCPIP, as previously reported (15). The lenses were homogenized in $0.1 \mathrm{M}$ phosphate-buffered saline (Ph 7.4) and mixed metaphosphoric acid for deproteinization. The lens homogenate was centrifuged; subsequently, the supernatant was titrated with DCPIP and the absorbance at $540 \mathrm{~nm}$ was measured by using a microplate reader infinite M1000 (Tecan Ltd.)

Measurement of cholesterol and triglyceride levels in plasma. Plasma sampels were separated by centrifugation of whole blood with heparin, and were stored at -80 before analysis. The total cholesterol and triglyceride levels were measured with SPOT-CHEM II and Kensin-2 strip (Arkray, Minneapolis, MN, USA).

Statistical analysis. Data were presented as the mean \pm standard error of the mean (SEM) of six different animals, and triplicate determinations were performed in each experiment. Statistical analysis of data was performed using one-way analysis of variance (ANOVA) with a post-hoc Tukey's multiple comparison test with SPSS software version 24 (BM Corp., Armonk, NY, USA). P<0.05 was considered to indicate a statistically significant difference.

\section{Results}

Roasting affects the anti-cataract ability of coffee brew. In this study, an obesity-related cataract model was induced by an HFD treatment. Using this model, we investigated whether the roasting process of coffee affected obesity-related cataract risks. The body weights of mice on day 0 and 49 is shown 

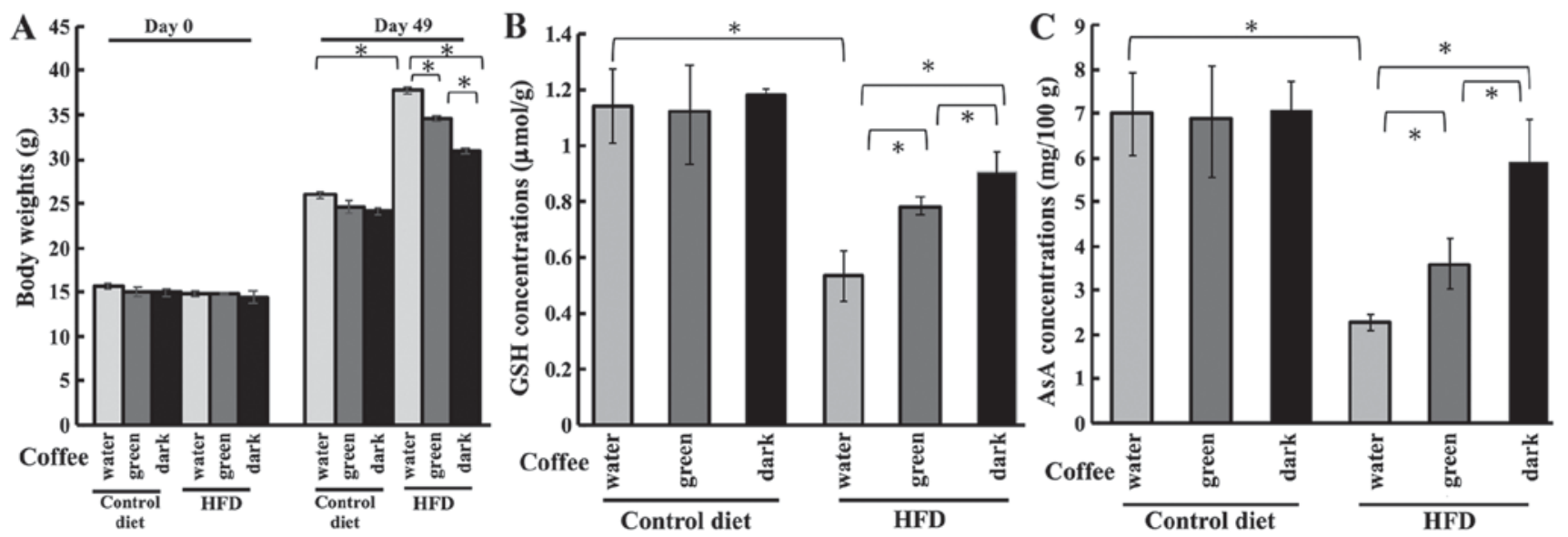

Figure 1. Effect of green beans coffee and roasting beans coffee in HFD-fed mice. (A) Body weights of HFD-fed mice treated with coffee brew from green beans or roasted beans on day 0 and day 49. (B) GSH and (C) AsA levels were measured for HFD-fed mice. GSH and AsA levels are measured by using DTNB and DCPIP, respectively. All results are expressed as the mean \pm standard error of the mean. Triplicate determinations were performed in each experiment ( $\mathrm{n}=6$ in each group). ${ }^{*} \mathrm{P}<0.05$. HFD, high-fat diet; GSH, glutathione; AsA, ascorbic acid; DTNB, dithionitrobenzene; DCPIP, 2,6-dichlorophenolindophenol.

in Fig. 1A. HFD-treated mice were significantly heavier than those fed the control diet; moreover, roasted coffee intake markedly reduced this increase in body weights. Subsequently, we investigated the effect of coffee intake on the antioxidant levels of the lens. In the control groups, there was no change in GSH concentration with the different brew treatment (Fig. 1B). The GSH concentration of the lens was significantly reduced to $0.53 \mu \mathrm{mol} / \mathrm{g}$ in the HFD group. However, this reduction in GSH was rescued with brew treatment: The GSH concentrations in the HFD-green beans group and HFD-roasted coffee group were 0.78 and $0.90 \mu \mathrm{mol} / \mathrm{g}$, respectively (Fig. 1B).

As the level of AsA in the lens is also a well-established cataract marker, we measured the AsA concentration in the lens in control- and HFD-treated mice. In the control groups, the AsA concentrations of the lens were unaffected by the treatments, whereas HFD-fed mice had significantly lower AsA levels. The AsA concentration in the lens of HFD-fed mice was $2.2 \mathrm{mg} / 100 \mathrm{~g}$ lens wet weight (Fig. 1C). Coffee intake ameliorated the HFD-induced AsA reduction, raising the concentrations to 3.60 and $5.90 \mathrm{mg} / 100 \mathrm{~g}$ lens wet weight for the green beans extract and dark-roasted coffee extract treatment groups, respectively (Fig. 1C). These results suggested that coffee consumption could prevent the reduction in the concentrations of GSH and AsA and that dark-roasted coffee was more effective than green-beans coffee for the treatment of HFD-induced obesity and cataract risk.

Coffee consumption ameliorated HFD-induced obesity and cataract risk. We administered three different concentrations of coffee extracts to HFD-fed mice to investigate the effect of coffee concentration effects on body weight and lens anti-oxidant compounds levels. The body weight increase in HFD-fed mice was abolished by treatment with $60 \%$ coffee extract, but not by $40 \%$ coffee extract (Fig. 2A). No significant changes were observed in the concentration of GSH in the controls with or without treatment, for all tested concentrations of coffee extract. Conversely, HFD-fed mice showed a marked reduction in GSH concentration without treatment, but coffee brew treatment in HFD-fed mice ameliorated GSH reduction
(Fig. 2B). Next, we measured AsA levels in HFD-fed mice to evaluate the anti-cataract effect of coffee. The concentration of AsA in the lens of control mice was unaffected by the different brew treatments. The AsA levels were significantly lower in HFD-fed mice, but coffee intake prevented this reduction in AsA (Fig. 2C). Collectively, these results indicated that coffee consumption could prevent HFD-induced reduction of GSH and AsA levels in a concentration-dependent manner.

Each coffee constituent affected the anti-cataract ability. Subsequently, we determined the constituent(s) of coffee directly responsible for the positive effects on cataract risk. The animals were treated with $60 \%$ coffee extract, $3.24 \mathrm{mM}$ caffeine, or $20.4 \mu \mathrm{M}$ pyrocatechol in each group. These concentrations represent the equivalent concentrations of the compounds in a $60 \%$ coffee brew (14). In the HFD groups, the body weight of mice on day 49 was reduced by coffee brew, caffeine, and pyrocatechol treatment (Fig. 3A). The equivalent treatments in the control group resulted in no changes in the GSH level in the lens. The levels of GSH in the lens were significantly reduced in HFD-fed animals; however, the reduction in GSH level in the lens was restored by treatment with coffee, caffeine, and pyrocatechol (Fig. 3B). Similarly, there were no changes in the levels of AsA in any treatment group in control-fed animals. The levels of AsA were also significantly reduced in the lens of HFD-treated animals. The co-administration of HFD and coffee brew, caffeine, or pyrocatechol rescued the reduction in AsA levels (Fig. 3C). These results suggested that caffeine and pyrocatechol in coffee brew may be the key constituents responsible for the preventing the reduction of GSH and AsA levels in the lens and reducing cataract risks of roasted coffee brew.

Coffee constituents affected the levels of plasma cholesterol and triglycerides. Subsequently, we quantified the levels of plasma cholesterol and triglycerides to evaluate the effects of coffee and coffee constituents on blood. Total cholesterol levels in HFD-fed mice were significantly higher compared to the levels in control diet group. Coffee brew, caffeine, or pyrocatechol treatment in HFD-fed mice group prevented 

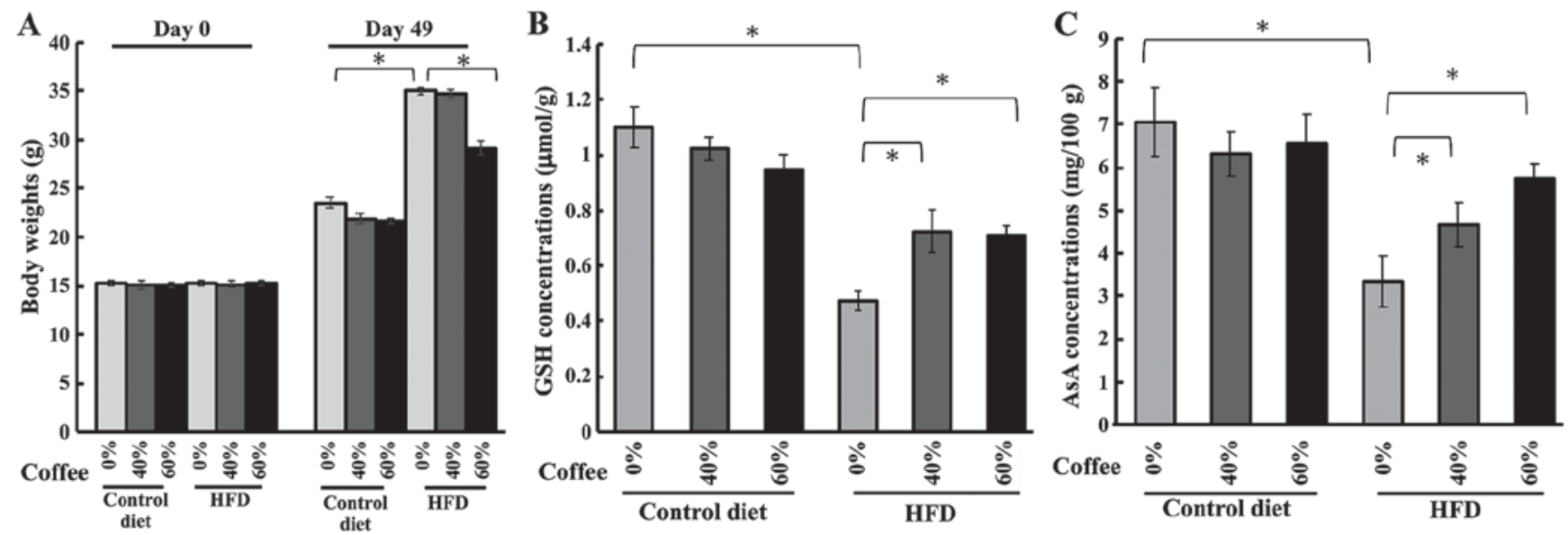

Figure 2. Measurement of body weight, and lens GSH or AsA level in HFD-treated mice with various concentrations of coffee brew. (A) Body weights of HFD-fed mice with various concentrations of dark-roasted coffee brew on day 0 and 49. Several concentrations of coffee brew were administered to HFD-fed mice and the levels of (B) GSH and (C) AsA were measured in the lens. All results are expressed as the mean \pm standard error of the mean. Triplicate determinations were performed in each experiment ( $\mathrm{n}=6$ in each group). ${ }^{*} \mathrm{P}<0.05$. HFD, high-fat diet; GSH, glutathione; AsA, ascorbic acid.

A

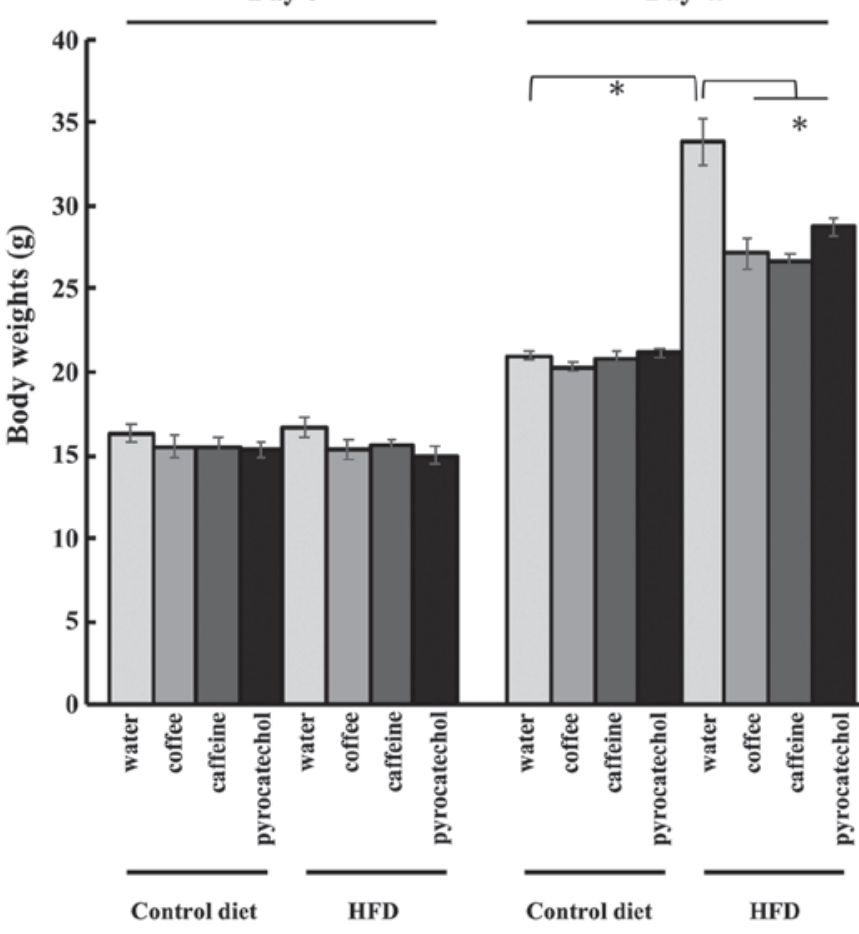

B

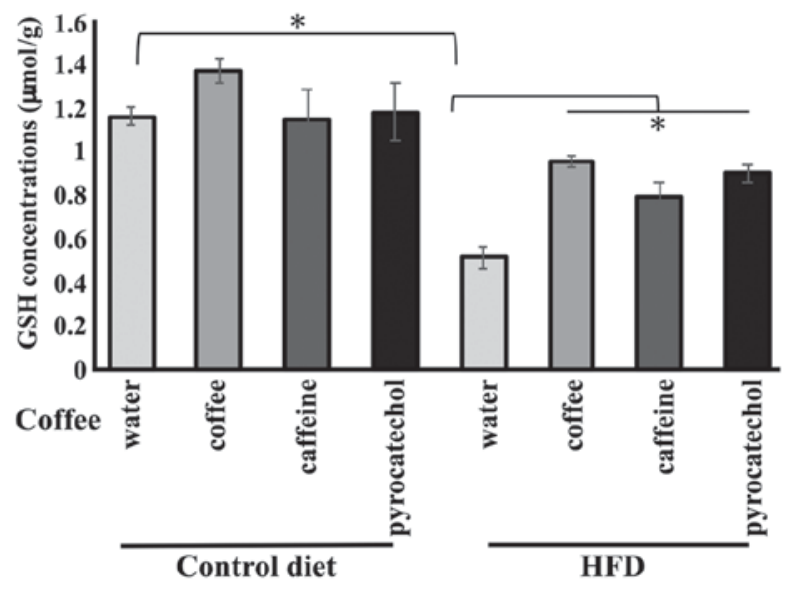

$\mathrm{C}$ ⿵冂丶

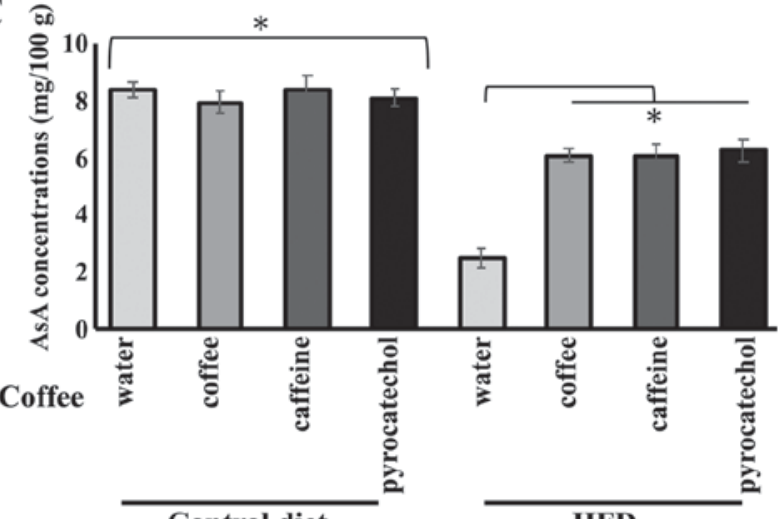

Control diet

HFD

Figure 3. Levels of GSH and AsA in lenses of HFD-fed mice treated with each coffee component. (A) Body weights of HFD-fed mice treated with coffee components on day 0 and 49. The concentrations of (B) GSH and (C) AsA in the lenses of HFD-fed mice administered each component. All results are expressed as the mean \pm standard error of the mean. Triplicate determinations were performed in each experiment ( $n=6$ in each group). ${ }^{*} \mathrm{P}<0.05$. HFD, high-fat diet; GSH, glutathione; AsA, ascorbic acid

elevation of total cholesterol (Fig. 4A). Next, the levels of plasma triglycerides in HFD-fed mice were measured, with or without administration of coffee constituents. Plasma triglycerides level was significantly higher in HFD-fed mice, however, increase in triglycerides level was negated by administration of coffee constituents (Fig. 4B).

\section{Discussion}

In HFD-induced obese animals, critical organs, such as the heart and kidney, are closely associated with elevated oxidative stress, as indicated by reduced levels of GSH, antioxidant enzymes such as glutathione peroxidase (GPx) and catalase 

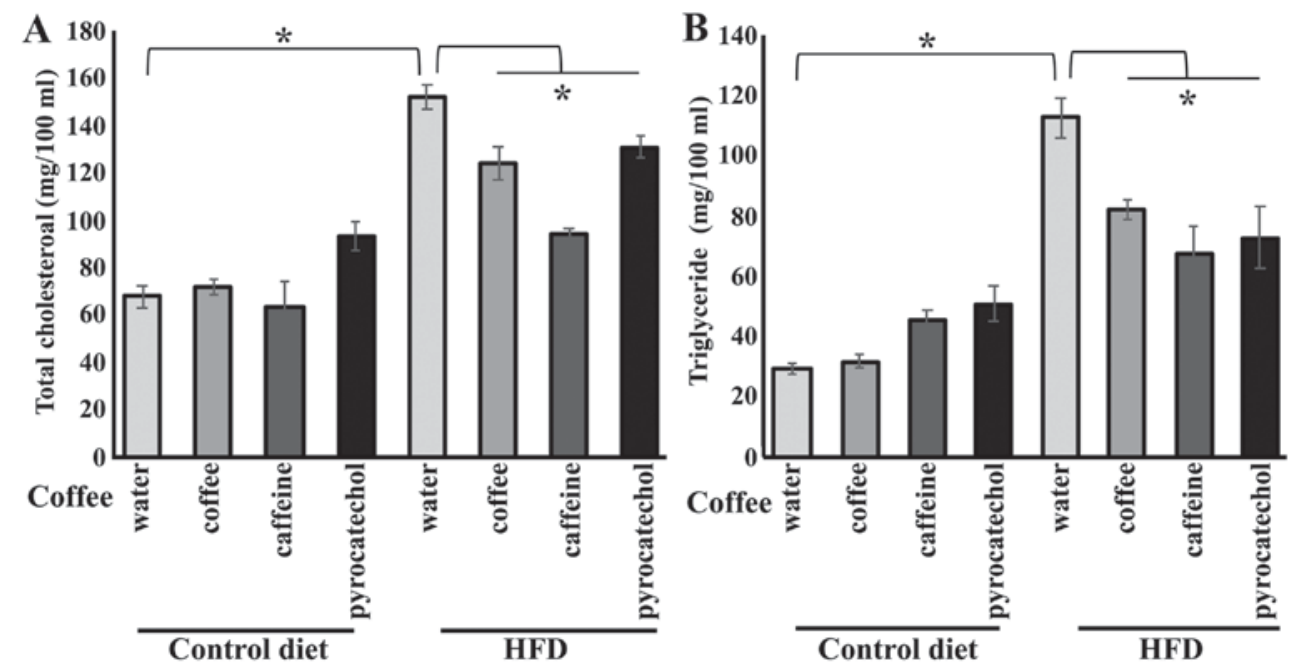

Figure 4. Levels of total cholesterol and triglycerides in HFD-fed mice treated with each coffee component. After seven weeks of HFD or control diet feeding, blood was collected and plasma samples were separated by centrifugation (A) Total cholesterol levels of HFD-fed mice treated with coffee components. (B) Triglyceride levels in plasma. All results are expressed as the mean \pm standard error of the mean ( $\mathrm{n}=6$ in each group). ${ }^{*} \mathrm{P}<0.05$. HFD, high-fat diet.

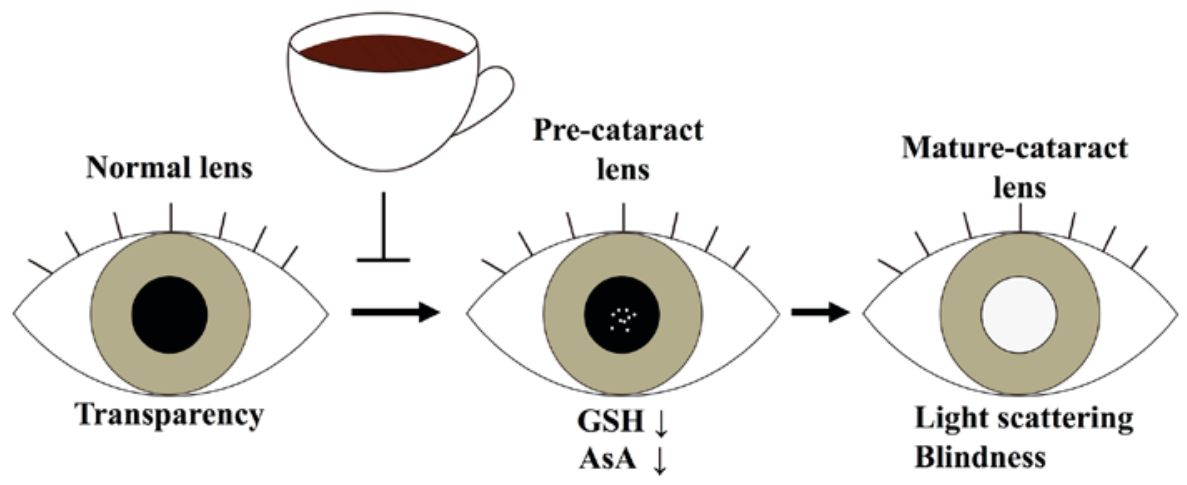

Figure 5. Inhibitory mechanism of cataract onset by coffee extract. Coffee brew intake could reduce cataract risks that ameliorates the HFD-induced GSH and AsA reduction. HFD, high-fat diet; GSH, glutathione; AsA, ascorbic acid.

(CAT), and elevated levels of malondialdehyde (MDA) and protein carbonyls (16). It is well established that the lens possesses an antioxidant defence system to minimize oxidative stress and protect against injury. GSH and AsA are the principal antioxidants in the lens, however, the levels of these compounds can become depleted with age, resulting in oxidative stress and, subsequently, mixed disulphide formation, protein aggregation, light scattering, and ultimately cataract formation. The preferred prevention strategy against cataracts is the maintenance of the antioxidant levels in the lens. In this report, we identified that coffee brew intake might reduce obesity-related cataract risks and maintain the normal GSH and AsA levels in the lens (Fig. 5).

It has been reported that $15-20 \%$ of obese rats between 350 and 450 days of age would develop spontaneous cataracts that were not apparent in age-matched controls (17). Another study on obese hyperglycaemic rats revealed bilateral cataracts in 240-day-old rats (18). In the current study, we evaluated the anti-cataract effects in HFD-treated mice for 7 weeks. In these mice, cataract formation was not observed using slit-lamp microscopy (data not shown); nonetheless, GSH and AsA levels in the lens were significantly lower than those of control mice (Figs. 1B and C, 2B and C, 3B and C). Previous studies have reported that reduction in concentrations of AsA and GSH predisposed to cataract and promoted the onset of mature cataract $(1,19,20)$. We have recognized that mice fed with HFD for 7 weeks presented with pre-cataract stage. Coffee brew, caffeine, and pyrocatechol were hypothesized to exert preventive effects against cataract by preventing reduction of AsA and GSH.

Caffeine, the major coffee component, is reported to act as an antioxidant to scavenge hydroxyl radical $(\mathrm{OH} \bullet)$ and inhibit oxidative stress (21). Pyrocatechol has also been reported to mimic anti-oxidant effects through the activation of Nrf2, heme-oxygenase-1 (HO-1), and NQO-1 (22). Therefore, the intake of coffee brew containing caffeine and pyrocatechol acts through several mechanisms to minimize cataract progression. Pyrocatechol in coffee is reported to be generated thought the roasting process and the significance of this roasting process is supported by our current data, which suggested that dark-roasted coffee brew rescued the HFD-induced reduction in GSH and AsA levels in the lens stronger than green beans coffee brew.

As age-related cataract progression in humans is very slow and therapeutics must be applied for a long duration to ameliorate or prevent the development of cataracts, it is vital to study the long-term safety of pharmacological therapies. In addition, it would be economically beneficial to find anti-cataract 
agents in the human diet that can be consumed daily. Here, we demonstrated that daily coffee consumption could reduce onset of obesity-related cataract risks to prevent the reduction of GSH and AsA levels in the lens.

\section{Acknowledgements}

Not applicable.

\section{Funding}

This study was supported by a grant from the Japan Society for the Promotion of Science KAKENHI (grant nos. 16K18957 and $15 \mathrm{~K} 00883$ ), which was awarded to YN and HT, from the All Japan Coffee Association and from the Keio Gijuku Fukuzawa Memorial Fund for the Advancement of Education and Research.

\section{Availability of data and materials}

The analyzed datasets generated during the study are available from the corresponding author on reasonable request.

\section{Authors' contribution}

YN and HT defined the research theme. YN, NN, MFT and HT designed the methods. YN, NI, NN, JO, NN, and MK performed the laboratory experiments. YN, NN, MFT and HT analyzed and interpreted the data. YN was major contributor in the writing of the manuscript.

\section{Ethics approval and consent to participate}

All animal experiments were approved by the Keio University Animal Research Committee [12048-(3)].

\section{Patient consent for publication}

Not applicable.

\section{Competing interests}

The authors declare that they have no competing interests.

\section{References}

1. Grey AC, Demarais NJ, West BJ and Donaldson PJ: A quantitative map of glutathione in the aging human lens. Int J Mass Spectrom (In Press)

2. Nakazawa Y, Oka M, Bando M and Takehana M: Hesperetin prevents selenite-induced cataract in rats. Mol Vis 21: 804-810, 2015.

3. Chasan-Taber L, Willett WC, Seddon JM, Stampfer MJ, Rosner B, Colditz GA, Speizer FE and Hankinson SE: A prospective study of carotenoid and vitamin A intakes and risk of cataract extraction in US women. Am J Clin Nutr 70: 509-516, 1999.
4. Isai M, Sakthivel M, Ramesh E, Thomas PA and Geraldine P: Prevention of selenite-induced cataractogenesis by rutin in Wistar rats. Mol Vis 15: 2570-2577, 2009.

5. Nakazawa Y, Nagai N, Ishimori N, Oguchi J and Tamura H: Administration of antioxidant compounds affects the lens chaperone activity and prevents the onset of cataracts. Biomed Pharmacother 95: 137-143, 2017.

6. Tan JS, Wang JJ and Mitchell P: Influence of diabetes and cardiovascular disease on the long-term incidence of cataract: The Blue Mountains eye study. Ophthalmic Epidemiol 15: 317-327, 2008.

7. Park S, Kim T, Cho SI and Lee EH: Association between cataract and the degree of obesity. Optom Vis Sci 90: 1019-1027, 2013.

8. Maki C, Funakoshi-Tago M, Aoyagi R, Ueda F, Kimura M, Kobata K, Tago K and Tamura H: Coffee extract inhibits adipogenesis in 3T3-L1 preadipocyes by interrupting insulin signaling through the downregulation of IRS1. PLoS One 12: e0173264, 2017.

9. Wu JN, Ho SC, Zhou C, Ling WH, Chen WQ, Wang CL and Chen YM: Coffee consumption and risk of coronary heart diseases: A meta-analysis of 21 prospective cohort studies. Int J Cardiol 137: 216-225, 2009.

10. Yu X, Bao Z, Zou J and Dong J: Coffee consumption and risk of cancers: A meta-analysis of cohort studies. BMC Cancer 11: 96, 2011.

11. Wang A, Wang S, Zhu C, Huang H, Wu L, Wan X, Yang X, Zhang $\mathrm{H}$, Miao R, He L et al: Coffee and cancer risk: A meta-analysis of prospective observational studies. Sci Rep 6: 33711, 2016.

12. Moura-Nunes N, Perrone D, Farah A and Donangelo CM: The increase in human plasma antioxidant capacity after acute coffee intake is not associated with endogenous non-enzymatic antioxidant components. Int J Food Sci Nutr 60 (Suppl 6): S173-S181, 2009.

13. Varma SD: Effect of coffee (caffeine) against human cataract blindness. Clin Ophthalmol 10: 213-220, 2016.

14. Ishimori N, Oguchi J, Nakazawa Y, Kobata K, Funakoshi-Tago M and Tamura $\mathrm{H}$ : Roasting enhances the anti-cataract effect of coffee beans: Ameliorating selenite-induced cataracts in rats. Curr Eye Res 42: 864-870, 2017.

15. Nakazawa Y, Oka M,Bando M, Inoue T and Takehana M: The role of ascorbic acid transporter in the lens of streptozotocin-induced diabetic rat. Biomed Prev Nutr 1: 43-48, 2011.

16. Sankhla M, Sharma TK, Mathur K, Rathor JS, Butolia V, Gadhok AK, Vardey SK, Sinha M and Kaushik GG: Relationship of oxidative stress with obesity and its role in obesity induced metabolic syndrome. Clin Lab 58: 385-395, 2012.

17. Reddy PY, Giridharan NV and Reddy GB: Activation of sorbitol pathway in metabolic syndrome and increased susceptibility to cataract in Wistar-Obese rats. Mol Vis 18: 495-503, 2012.

18. McCaleb ML and Sredy J: Metabolic abnormalities of the hyperglycemic obese Zucker rat. Metabolism 41: 522-525, 1992.

19. Kisic B, Miric D, Zoric L, Ilic A and Dragojevic I: Antioxidant capacity of lenses with age-related cataract. Oxid Med Cell Longev 2012: 467130, 2012.

20. Pescosolido N, Barbato A, Giannotti R, Komaiha C and Lenarduzzi F: Age-related changes in the kinetics of human lenses: Prevention of the cataract. Int J Ophthalmol 18: 1506-1517, 2016.

21. Varma SD, Hegde KR and Kovtun S: UV-B-induced damage to the lens in vitro: Prevention by caffeine. J Ocul Pharmacol Ther 24: 439-444, 2008.

22. Sirota R, Gibson D and Kohen R: The role of the catecholic and the electrophilic moieties of caffeic acid in Nrf2/Keap1 pathway activation in ovarian carcinoma cell lines. Redox Biol 4: 48-59, 2015. 